\title{
Fruit and vegetable intake and risk of acute coronary syndrome
}

\author{
Louise Hansen $^{1}$, Lars O. Dragsted ${ }^{2}$, Anja Olsen ${ }^{1}$, Jane Christensen ${ }^{1}$, Anne Tjønneland ${ }^{1}$, Erik B. Schmidt ${ }^{3}$ \\ and Kim Overvad ${ }^{3,4}$ \\ ${ }^{1}$ Danish Cancer Society, Institute of Cancer Epidemiology, Strandboulevarden 49, DK-2100 Copenhagen, Denmark \\ ${ }^{2}$ Department of Human Nutrition, Faculty of Life Sciences, Copenhagen University, Frederiksberg, Denmark \\ ${ }^{3}$ Department of Cardiology, Centre for Cardiovascular Research, Aalborg Hospital, Aarhus University Hospital, Aalborg, \\ Denmark \\ ${ }^{4}$ Department of Epidemiology, Institute of Public Health, Aarhus University, Aarhus, Denmark
}

(Received 9 June 2009 - Revised 5 January 2010 - Accepted 11 January 2010 - First published online 24 February 2010)

\begin{abstract}
Prospective epidemiological studies have reported that a higher fruit and vegetable intake is associated with a lower risk of CHD. The aim of the present study was to examine associations between fruit and vegetable consumption, in particular the subgroupings citrus fruits, apples and cruciferous vegetables, and the risk of acute coronary syndrome (ACS). During a median follow-up of 7.7 years, 1075 incident ACS cases were identified among 53383 men and women, aged 50-64 years at recruitment into the Diet, Cancer and Health cohort study in $1993-7$. Fruit and vegetable intake was estimated from a validated FFQ, and ACS incidence rate ratios (IRR) were estimated using Cox proportional hazards models. Overall, a tendency towards a lower risk of ACS was observed for both men and women with higher fruit and vegetable consumption. For men, we found an inverse association for apple intake (IRR per $25 \mathrm{~g} / \mathrm{d}$ : 0.97; $95 \%$ CI 0.94, 0.99). This association was also seen among women, albeit borderline significant. However, a higher risk was seen among women with higher fruit juice intake (IRR per $25 \mathrm{~g} / \mathrm{d}: 1.04 ; 95 \%$ CI $1.00,1.08)$. The present results provide some support for previously observed inverse associations between fresh fruit intake, particularly apples, and ACS risk.
\end{abstract}

Fruits: Vegetables: CHD

A higher consumption of fruits and vegetables has been associated with a lower risk of CHD in several epidemiological studies $^{(1-5)}$. Furthermore, two recent meta-analyses have reported an inverse association between fruit and vegetable consumption and the risk of $\mathrm{CHD}^{(6,7)}$.

Several mechanisms may explain the possible cardioprotective qualities of fruit and vegetables, including those concerning antioxidants, dietary fibre and cholesterol-lowering effects $^{(8)}$. The biological mechanisms behind these effects are not entirely clear and are likely to be both multi-fold and interconnected. Most studies have not examined the effects of specific types of fruits and vegetables; in addition, studies frequently examine proxies of fruit and vegetable intake, such as antioxidant intake ${ }^{(2)}$. Furthermore, CVD has often been investigated without discriminating between CHD and cerebrovascular disease.

In the present study, we focused solely on CHD events. Thus, we investigated the associations between total fruit and vegetable intake and of specific fruit and vegetable groupings in relation to the risk of acute coronary syndrome (ACS), which is the composite of unstable angina pectoris, myocardial infarction and cardiac arrest. Investigated food items were selected a priori, and two fruit groupings of particular interest were citrus fruits, as lowered risk for citrus fruits was seen in the PRIME (Prospective Epidemiological Study of Myocardial Infarction) study ${ }^{(1)}$ as well as apples due to their possible cholesterol-lowering properties ${ }^{(9)}$. Furthermore, fruit juices were examined separately, as they lack fibre, are less satiating and have a high sugar content. Juice has in the Nurses' Health Study been linked to a higher risk of diabetes mellitus, which is an important risk factor for $\mathrm{CHD}^{(10)}$. Cruciferous vegetables were also of particular interest, as they have been found to be inversely associated with the risk of $\mathrm{CHD}^{(2,11)}$, presumably due to their content of isothiocyanates.

\section{Methods}

The present study is based on data from the prospective Danish Diet, Cancer and Health cohort study. A total of 57053 men and women were enrolled into the cohort in 1993-7 and were included if they fulfilled the following criteria: aged between 50-64 years, born in Denmark and no previous cancer diagnosis in the Danish Cancer Registry.

A detailed FFQ and lifestyle questionnaire were filled in by each participant. Development and validation of the FFQ are 
described elsewhere ${ }^{(12,13)}$. The FFQ contained questions regarding 192 food and beverage items and was developed to obtain information on the participants' habitual diet during the preceding year. Biological and anthropometric measurements were taken including height (m) and weight $(\mathrm{kg})$, from which BMI was calculated $\left(\mathrm{kg} / \mathrm{m}^{2}\right)$. A thorough description of data collection has been published previously $^{(14)}$.

\section{Participant exclusions}

The present cohort was originally established to investigate cancer; thus, individuals with a prevalent diagnosis of cancer at study enrolment ( $n$ 564) were excluded from the present study. While the present study concerns heart disease, these individuals are always removed from studies done in the cohort. Furthermore, a previous diagnosis of ACS at baseline was cause for exclusion ( $n$ 1013). Also, individuals with a diagnosis of diabetes mellitus at baseline as determined from the National Diabetes Register were excluded due to potential effect modification, which could not be explored due to low numbers, as well as potential dietary changes after diagnosis and treatment ( $n$ 1106). After exclusion due to ACS or diabetes mellitus, 54370 individuals remained in the study. Further reasons for exclusions included missing medical information ( $n$ 123), an incomplete lifestyle questionnaire ( $n$ 42) and missing information on potential confounders ( $n$ 759) or dietary variables ( $n$ 63). A total of 53383 individuals were ultimately included in the study.

\section{Follow-up and ascertainment of acute coronary syndrome cases}

The participants in the cohort were linked to the Central Population Registry for information on vital status and emigration. Information on ACS incidence was obtained by linkage of the Central Population Registry number of each participant to the National Patient Registry and the Cause of Death Registry. Each individual was followed for primary ACS occurrence from date of entry until censoring, which included date of event (fatal or non-fatal ACS), death of another cause, migration or 31 December 2003. Incident cases of fatal or non-fatal ACS in the cohort were identified and defined according to the International Classification of Diseases (ICD) eighth revision (ICD-8 diagnosis codes 410-410.99 and 427.27) until 31 December 1993 and subsequently by ICD tenth revision (ICD-10 diagnosis codes I20.0, I21.0-I21.9 and I46.0-I46.9). There have been considerable changes in the definition of myocardial infarction since 2000 and therefore since the beginning of the Danish Diet, Cancer and Health study, and in order to minimise potential discrepancies in ACS ascertainment, each case was individually validated through review of medical records. In fact, more than $80 \%$ of the cases were diagnosed using very sensitive biomarkers (troponins and creatine kinase $\mathrm{MB}$ (CK-MB)). When cases ascertained before or after 2000 were compared, no significant differences in positive predictive value were seen, thus ensuring correct ACS diagnoses ${ }^{(15)}$. During a median follow-up of 7.7 years, 1075 cases were identified.

\section{Dietary assessment}

Intake of fruits and vegetables was estimated based on the FFQ completed at baseline. Daily intake was calculated for each participant using the software program FoodCalc ${ }^{(16)}$ and specifically developed standardised recipes and portion sizes. For fruit, only intake of fresh fruit (as indicated on the FFQ) was examined, while vegetable intake also included estimated contributions from recipes. This was done to ensure maximal validity of the fruit groupings, since contributions from recipes for fruit intake are likely to be minimal. The groupings used for fruit were: total fruits, citrus fruits (consisting of oranges, mandarins, grapefruits), other fruits (pears, bananas, nectarines/peaches, strawberries, kiwis, melons), apples (all sorts) and fruit juice (orange, grape). For vegetables, groupings were: total vegetables, cruciferous vegetables (broccoli, cauliflower, Brussels sprouts, kale, cabbages, pointed cabbages) and other vegetables (including types of leafy vegetables, fruiting vegetables, root vegetables, mushrooms, onions and stalk vegetables/sprouts).

\section{Statistical analyses}

Analyses of the associations between fruit and vegetable intake and the incidence rate ratios (IRR) of ACS were based on Cox proportional hazards models (including time-dependent variables) using age as the time axis to ensure that the estimation procedure was based on comparisons of individuals at the same age. Time-under-study was included as the time-dependent variable and was modelled by a linear spline with a boundary at 1,2 and 3 years after entry into the cohort study to allow the rate to change with time. The proportional hazards assumption was for each categorical variable evaluated graphically using a Kaplan-Meier plot. Two-sided $95 \%$ CI for the IRR were calculated on the basis of Wald's test of the Cox regression parameter, i.e. on the $\log$ rate ratio scale. Analyses were performed separately for men and women.

All quantitative variables were entered linearly into Cox models after linear spline testing, as this is biologically more plausible than the step functions corresponding to categorisation; furthermore, this approach increases the power of the analysis ${ }^{(17)}$. The exposure variables were included as a linear variable in a specific unit $(\mathrm{g} / \mathrm{d})$, which was determined based on the interquartile range and a realistic difference in consumption. Therefore, $100 \mathrm{~g} / \mathrm{d}$ for total fruits and total vegetables and $25 \mathrm{~g} / \mathrm{d}$ for subgroups of fruits and vegetables were used ( $25 \mathrm{~g}$ corresponds approximately to a quarter of a medium-sized apple). Quartile analyses were also performed, although tertiles were used for apples to account for the categories provided by the questionnaire. Categories were based on the baseline intake in the cohort for men and women, respectively.

Age-adjusted IRR (presented as crude) were examined along with two multivariable models. In the first multivariable model, intake was mutually adjusted as well as adjusted for baseline values of potential risk factors for ACS: BMI (linear), length of school education ( $\leq 7$ years, $8-10$ years and $>10$ years), smoking (never, former, current (1-14, $15-25$ or $>25 \mathrm{~g}$ tobacco per $\mathrm{d}$ )), alcohol intake (linear spline with a boundary at $10 \mathrm{~g} / \mathrm{d}$ ), alcohol abstainers and 
physical activity ( $\leq$ or $>3.5 \mathrm{~h}$ per week and non-active), intake of saturated fats $(\mathrm{g} / \mathrm{d})$ and of whole grains $(\mathrm{g} / \mathrm{d})$. The second multivariable model was additionally adjusted for total serum cholesterol levels $(\mathrm{mmol} / \mathrm{l})$ and systolic blood pressure ( $\mathrm{mmHg}$ ).

The PHREG procedure in SAS (release 9.1; SAS Institute Inc., Cary, NC, USA) on a TextPad platform was used for the statistical analyses on incidence, while the LIFETEST procedure in SAS was used to test the proportional hazards assumption.

\section{Results}

The study population consisted of $53 \%$ ( $n$ 28318) women. However, a substantially larger proportion of the cases occurred in men $(n 820 ; 76 \%)$. Table 1 shows selected CHD risk factors by quartile of total fruit and vegetable intake for men and women. Men with the highest intakes of fruits and vegetables were more likely to have a higher education, to be physically active and to have slightly lower total cholesterol levels. Also, they had a lower alcohol and tobacco consumption, higher whole-grain consumption, but also slightly higher consumption of saturated fats than those with the lowest intakes of fruits and vegetables. Overall, the same distribution pattern was seen for women.

Intakes of total fruits and vegetables and of citrus fruits, other fruits, fruit juice, apples, other vegetables and cruciferous vegetables are presented in Table 2. In general, men had a lower intake of fruits and vegetables than women. Male cases had a lower intake of all groupings of fruits and vegetables than the cohort. Fruit juice intake, however, was identical among cases and the cohort. The same pattern was evident for women except for apples, where female cases and the cohort had the same median apple intake of $54 \mathrm{~g} / \mathrm{d}$.

Intake of fruits and vegetables combined per $100 \mathrm{~g} / \mathrm{d}$ was borderline significantly associated with a lower risk of ACS for both men and women in the continuous analyses (results not shown in a Table). For men, the crude IRR was 0.91 (95\% CI 0.87, 0.95), while for women the estimate was 0.88 (95\% CI $0.82,0.94$ ). However, the estimates were attenuated after multivariable adjustment and were borderline significant (for men, IRR 0.97 (95\% CI 0.93, 1.01) and for women, IRR 0.95 (95\% CI 0.89, 1.01)).

Table 3 presents the association between fruit consumption and the risk of ACS for both men and women. Overall, the crude models indicated inverse associations between intake of total fruits as well as of the various groupings in relation to ACS risk. However, after multivariable adjustment, only few associations persisted. Higher total fruit intake was associated with a non-significant $4 \%$ lower risk of ACS for men (IRR per $100 \mathrm{~g} / \mathrm{d}: 0.96$; $95 \%$ CI 0.91, 1.02). Apple intake was likewise associated with a lower risk of ACS (IRR per $25 \mathrm{~g} / \mathrm{d}: 0.97 ; 95 \%$ CI $0.94,0.99$ ). The categorical estimates showed lower risk for those in the top tertile of apple intake; the IRR for the top tertile was $0.78(95 \% \mathrm{CI}$ $0.64,0.95)$ in the multivariable-adjusted analyses. No associations were seen for intake of citrus or other fruits.

Similar results were seen among women (Table 3). Crude models indicated inverse associations between fruit groupings and ACS risk, but most associations did not persist after adjustment. However, intake of total fruits was borderline significantly associated with a lower risk of ACS (IRR per $100 \mathrm{~g} / \mathrm{d}: 0.93 ; 95 \%$ CI $0.85,1.01)$, and apple intake was associated with a $3 \%$ lower risk of ACS (IRR per $25 \mathrm{~g} / \mathrm{d}$ : $0.97 ; 95 \%$ CI $0.93,1.01)$. No associations were seen for intake of citrus or other fruits. Intake of fruit juice was associated with a $4 \%$ higher risk of ACS (IRR per $25 \mathrm{~g} / \mathrm{d}$ : $1.04 ; 95 \%$ CI 1.00, 1.09). No fruit groupings were associated with statistically significant results in the adjusted models when examined categorically.

Table 4 presents the associations between total vegetable intake and ACS risk for both men and women. For total and cruciferous vegetable intake among men, significantly lower risks were seen in the crude models, but these did not persist after adjustment (IRR per $100 \mathrm{~g} / \mathrm{d}$ for total vegetables: 0.98; $95 \%$ CI $0.90,1.07$; IRR per $25 \mathrm{~g} / \mathrm{d}$ for cruciferous vegetables: 1.05 ; $95 \%$ CI $0.91,1.22$ ). When the associations were examined categorically, intake of total vegetables was associated with lower risk in the top quartile of intake, but this estimate was no longer significant after adjustment (IRR 0.93; $95 \%$ CI $0.75,1.16)$. Among women, total and cruciferous vegetable intakes were both associated with significantly lower risk in the crude estimates, but, again, these results were attenuated and non-significant in the adjusted models (IRR per $100 \mathrm{~g} / \mathrm{d}$ for total vegetables: 0.97 ; $95 \%$ CI $0.84,1.13$; IRR per $25 \mathrm{~g} / \mathrm{d}$ for cruciferous vegetables: $0.99 ; 95 \%$ CI $0.76,1.29)$. No associations were seen for intake of other vegetables for either men or women.

\section{Discussion}

We observed an inverse association between apple intake and risk of ACS for both men and women. Furthermore, fruit juice consumption was associated with a higher ACS risk among women. Total fruit intake also indicated a protective association with ACS, but the associations were borderline significant. For intake of vegetables no consistent associations were observed.

The nearly complete follow-up (99.6\%) of this populationbased prospective study, as well as the large number of cases, are among the strengths of the present study. Exposure information was obtained before diagnosis of ACS, and the detailed information regarding fruit and vegetable intake made it possible to look at groupings of fruits and vegetables of interest; this was done using both linear and categorical estimates. It was possible to examine apples as a separate group, which is highly relevant, as this fruit is a major contributor to fruit intake in Denmark. Apple intake was specified in the FFQ, and intake is thus based on fresh fruit intake and not estimated from recipes. Furthermore, the individual validation of all ACS cases ensured that only those diagnoses that truly were an ACS event were included; thus, information bias is not likely to have affected the study results. A limitation of the present study is potential over-reporting of fruits and vegetables. It is conceivable that intake of fruits and vegetables were over-reported, as they are often perceived to be healthpromoting, but it seems unlikely that some groupings were over-reported in the FFQ to a higher degree than others. Information on all fruit and vegetable types was collected using identical questions with the same twelve possible answer categories. It is, however, possible that intake data on fruit are more valid than those of vegetables, as only intake from 
Table 1. Baseline characteristics for the Diet, Cancer and Health cohort (men and women) for selected CHD risk factors according to quartiles (Q) of total fruit and vegetable intake (Median values and 5th and 95th percentiles within each category or percentages)

\begin{tabular}{|c|c|c|c|c|c|c|c|c|c|c|c|c|c|c|c|c|c|c|c|c|}
\hline \multirow{4}{*}{$\begin{array}{l}\text { Range of intake }(\mathrm{g} / \mathrm{d}) \\
\text { Variable }\end{array}$} & \multicolumn{10}{|c|}{ Men } & \multicolumn{10}{|c|}{ Women } \\
\hline & \multirow[b]{3}{*}{ Mediar } & $\begin{array}{l}\text { Cohort } \\
25 \text { 065) }\end{array}$ & \multicolumn{2}{|r|}{ Q1 } & \multicolumn{2}{|r|}{ Q2 } & \multicolumn{2}{|c|}{ Q3 } & \multicolumn{2}{|r|}{ Q4 } & \multicolumn{2}{|c|}{$\begin{array}{c}\text { Cohort } \\
\text { (n 28 318) }\end{array}$} & \multicolumn{2}{|r|}{ Q1 } & \multicolumn{2}{|r|}{ Q2 } & \multicolumn{2}{|r|}{ Q3 } & \multicolumn{2}{|r|}{ Q4 } \\
\hline & & & $>0 \mathrm{t}$ & to $\leq 180$ & $>180$ & to $\leq 273$ & $>273$ & to $\leq 392$ & & $>392$ & \multirow[b]{2}{*}{ Median } & \multirow[b]{2}{*}{ n $5-95 \%$} & \multicolumn{2}{|c|}{$>0$ to $\leq 237$} & \multicolumn{2}{|c|}{$>237$ to $\leq 350$} & \multicolumn{2}{|c|}{$>350$ to $\leq 497$} & \multicolumn{2}{|c|}{$>497$} \\
\hline & & $5-95 \%$ & Median & n $5-95 \%$ & Median & n $5-95 \%$ & Median & $5-95 \%$ & Median & in $5-95 \%$ & & & Median & $5-95 \%$ & Median & n $5-95 \%$ & Median & $5-95 \%$ & Median & n $5-95 \%$ \\
\hline Age (years) & 55 & $50-64$ & 56 & $50-64$ & 55 & $50-64$ & 55 & $50-64$ & 55 & $50-64$ & 56 & $50-64$ & 56 & $50-64$ & 56 & $50-64$ & 56 & $50-64$ & 56 & $50-64$ \\
\hline \multicolumn{21}{|l|}{ Education (\%) } \\
\hline$\leq 7$ years & \multicolumn{2}{|r|}{34} & \multicolumn{2}{|r|}{44} & \multicolumn{2}{|r|}{34} & \multicolumn{2}{|r|}{30} & & 29 & & 31 & & 38 & & 32 & & 27 & & 27 \\
\hline $8-10$ years & & 42 & & 41 & & 45 & & 42 & & 39 & & 50 & & 50 & & 51 & & 51 & & 50 \\
\hline$>10$ years & & 24 & & 15 & & 21 & & 28 & & 32 & & 19 & & 12 & & 17 & & 22 & & 23 \\
\hline BMI $\left(\mathrm{kg} / \mathrm{m}^{2}\right)$ & 26 & $21-33$ & 26 & $21-33$ & 26 & $21-33$ & 26 & $22-33$ & 26 & $22-33$ & 25 & $20-34$ & 25 & $20-34$ & 25 & $20-33$ & 25 & $20-34$ & 25 & $20-34$ \\
\hline Smoking status (\%) & & & & & & & & & & & & & & & & & & & & \\
\hline Never & & 26 & & 19 & & 27 & & 28 & & 31 & & 44 & & 35 & & 45 & & 47 & & 49 \\
\hline Former & & 34 & & 27 & & 32 & & 38 & & 41 & & 23 & & 18 & & 24 & & 25 & & 28 \\
\hline Current & & & & & & & & & & & & & & & & & & & & \\
\hline $1-14 \mathrm{~g}$ tobacco/d & & 11 & & 10 & & 11 & & 11 & & 10 & & 15 & & 17 & & 15 & & 15 & & 13 \\
\hline $15-24 \mathrm{~g}$ tobacco/d & & 17 & & 25 & & 19 & & 15 & & 11 & & 15 & & 25 & & 14 & & 12 & & 9 \\
\hline $25+\mathrm{g}$ tobacco/d & & 11 & & 19 & & 11 & & 8 & & 7 & & 3 & & 5 & & 2 & & 1 & & 1 \\
\hline Alcohol intake $(\mathrm{g} / \mathrm{d})^{\star}$ & 20 & $2-81$ & 22 & 2-91 & 20 & $3-82$ & 20 & $3-70$ & 18 & $2-67$ & 10 & $1-42$ & 10 & $1-53$ & 10 & $1-43$ & 10 & $1-40$ & 8 & $1-37$ \\
\hline Abstainers (\%) & 20 & 1.7 & 26 & $2 \cdot 2$ & 20 & 1.5 & $<0$ & 1.3 & 10 & 1.9 & & 2.6 & & 3.6 & & $2 \cdot 1$ & & 1.9 & & 2.9 \\
\hline Physical activity (\%) & & & & & & & & & & & & & & & & & & & & \\
\hline Not physically active & & 21 & & 31 & & 22 & & 17 & & 14 & & 16 & & 23 & & 16 & & 13 & & 11 \\
\hline$\leq 3.5 \mathrm{~h}$ per week & & 44 & & 44 & & 46 & & 47 & & 41 & & 47 & & 50 & & 50 & & 47 & & 41 \\
\hline$>3.5 \mathrm{~h}$ per week & & 35 & & 25 & & 32 & & 36 & & 45 & & 37 & & 27 & & 34 & & 40 & & 48 \\
\hline $\begin{array}{l}\text { Total cholesterol } \\
(\mathrm{mmol} / \mathrm{l})\end{array}$ & 5.9 & $4 \cdot 3-7 \cdot 9$ & 6.0 & $4 \cdot 4-8.0$ & 5.9 & $4 \cdot 3-7 \cdot 9$ & 5.9 & $4 \cdot 3-7 \cdot 9$ & 5.8 & $\begin{array}{ll}3 & 4.2-7.8\end{array}$ & 6.2 & $4.5-8.4$ & 6.3 & $4.6-8.5$ & 6.2 & $4.5-8.5$ & $6 \cdot 1$ & $4.5-8.3$ & 6.1 & $4.5-8.3$ \\
\hline $\begin{array}{l}\text { Systolic blood } \\
\text { pressure }(\mathrm{mmHg})\end{array}$ & 140 & $114-176$ & 141 & $115-179$ & 140 & $113-176$ & 139 & $114-176$ & 140 & $114-176$ & 136 & $106-175$ & 136 & $107-176$ & 135 & $106-176$ & 135 & $106-172$ & 135 & $106-174$ \\
\hline $\begin{array}{l}\text { Saturated fat } \\
\text { intake }(\mathrm{g} / \mathrm{d})\end{array}$ & 36 & $20-61$ & 34 & $19-57$ & 36 & $20-59$ & 37 & $20-61$ & 38 & $20-65$ & 28 & $15-48$ & 26 & $14-48$ & 27 & $15-46$ & 28 & $15-49$ & 29 & $14-52$ \\
\hline $\begin{array}{l}\text { Whole-grain } \\
\text { intake }(\mathrm{g} / \mathrm{d})\end{array}$ & 130 & $41-267$ & 112 & $22-242$ & 130 & $42-263$ & 144 & $58-267$ & 163 & $61-300$ & 113 & $31-221$ & 98 & $25-213$ & 110 & $33-214$ & 116 & $42-234$ & 124 & $42-252$ \\
\hline
\end{tabular}

${ }^{*}$ Alcohol intake among drinkers. 
Table 2. Baseline intake of fruits and vegetables for the Diet, Cancer and Health cohort (men and women) and acute coronary syndrome cases (Median values with 5th and 95th percentiles within each category)

\begin{tabular}{|c|c|c|c|c|c|c|c|c|}
\hline \multirow[b]{3}{*}{ Variable $(g / d)$} & \multicolumn{4}{|c|}{ Men } & \multicolumn{4}{|c|}{ Women } \\
\hline & \multicolumn{2}{|c|}{ Cohort ( $n 25$ 065) } & \multicolumn{2}{|c|}{ Cases $(n 820)$} & \multicolumn{2}{|c|}{ Cohort (n 28318$)$} & \multicolumn{2}{|c|}{ Cases ( $n$ 255) } \\
\hline & Median & $5-95 \%$ & Median & $5-95 \%$ & Median & $5-95 \%$ & Median & $5-95 \%$ \\
\hline Total fruit and vegetables (excluding juice) & 273 & $84-646$ & 247 & $65-598$ & 350 & $114-789$ & 305 & $84-710$ \\
\hline Total fruit (excluding juice) & 112 & $15-411$ & 97 & $12-401$ & 168 & $28-506$ & 149 & $19-439$ \\
\hline Apples & 54 & $2-313$ & 18 & $2-125$ & 54 & $2-313$ & 54 & $2-125$ \\
\hline Citrus fruit & 11 & $2-101$ & 9 & $2-101$ & 20 & $3-118$ & 17 & $2-111$ \\
\hline Other fruit & 35 & $7-162$ & 32 & $6-167$ & 61 & $11-240$ & 51 & $9-223$ \\
\hline Fruit juice $^{*}$ & 8 & $0-100$ & 8 & $0-100$ & 9 & $0-101$ & 8 & $0-250$ \\
\hline Total vegetables (excluding juice) & 148 & $47-328$ & 129 & $37-314$ & 167 & $50-371$ & 139 & $42-331$ \\
\hline Cruciferous vegetables & 14 & $2-43$ & 13 & $2-42$ & 16 & $3-45$ & 13 & $2-38$ \\
\hline Other vegetables & 131 & $41-296$ & 115 & $33-287$ & 149 & $45-340$ & 121 & $37-296$ \\
\hline
\end{tabular}

* The range of fruit juice intake is among consumers but has been rounded off to the nearest whole number. Minimum intake for both sexes was $0.01 \mathrm{~g}$ fruit juice/d.

fresh fruits and juice (i.e. what was actually reported in the FFQ) was included for analysis, whereas for vegetables, intake estimated from recipes was also included.

The associations were examined separately by sex, as men have a higher risk of $\mathrm{CHD}$, and risk factors differ to some extent between the sexes. Furthermore, a range of potential confounders, primarily chosen based on literature review, were adjusted for in multivariable models. Estimates changed markedly, when the analyses were mutually and multivariable adjusted. Specifically, we adjusted for various known risk factors of CHD as well as dietary factors possibly associated with fruit and vegetable intake. Residual confounding can thus not be excluded, and more detailed control may further attenuate the observed associations. In a second multivariable model, we adjusted for total cholesterol and systolic blood pressure, as both are risk factors for CHD but are also possible intermediate factors in the causal pathway between fruit and vegetable intake and ACS risk. As mentioned earlier, it is hypothesised that an effect on ACS risk from, for example, apples might be due to a cholesterol-lowering effect. However, the association between intake of apples and risk of ACS was also present after control for the potential intermediate variables, indicating that other mechanisms may be important in explaining the observed association.

Overall, the present results indicate associations that are consistent with those observed in similar cohort studies. Two recent meta-analyses have summarised the current evidence from all eligible cohort studies on risk of CHD and fruit and vegetable intake, and both reported an inverse association. In the meta-analysis by Dauchet et al. ${ }^{(6)}$, a relative risk of 0.96 (95\% CI $0.93,0.99)$ per portion per $d$ for fruit and vegetable intake overall was found, and a more marked decrease for fruit consumption alone was seen (relative risk 0.93; $95 \%$ CI 0.89, 0.96). Similarly, He et al. ${ }^{(7)}$ found a pooled relative risk of $0.83(95 \% \mathrm{CI} 0.77,0.89)$ for an intake of fruit and vegetables more than five servings/d.

In the present study, apples were found to be associated with a lower risk of ACS. Likewise, a lower risk of CHD mortality in association with apple intake was seen in a Finnish cohort study by Knekt et al. ${ }^{(18)}$ where the relative risks between the highest and lowest quartiles of apple intake were 0.57 (95\% CI $0.36,0.91)$ and $0.81(95 \% \mathrm{CI}$ $0.61,1.09$ ) for women and men, respectively ${ }^{(18)}$. An American cohort study including 35000 women further found that the phytochemicals catechin and epicatechin from apples and tea were strongly inversely related to CHD mortality ${ }^{(19)}$. The results seen for apples in the present study could potentially be due to a lowering of cholesterol, as apples have been found to have serum cholesterol-lowering properties in several rodent studies ${ }^{(20-22)}$. Human intervention studies are sparse; however, one study investigating the effects of fresh apple consumption on cholesterol levels found reduced total plasma cholesterol and increased HDL-cholesterol. On average, a reduction exceeding $10 \%$ of their starting plasma cholesterol level was observed among the study participants $^{(23)}$. Furthermore, a Japanese intervention study on intake of supplements containing apple polyphenols found lowered levels of total and LDL-cholesterol ${ }^{(9)}$.

The other groupings that were investigated separately in the present study were cruciferous vegetables and citrus fruits. Cruciferous vegetables have a high content of isothiocyanates and have been found in other studies to be inversely associated with $\mathrm{CHD}^{(2,11)}$. In the present study, no consistent associations were observed, which may in part be due to the rather low intake of cruciferous vegetables in the cohort. Citrus fruits have in the PRIME (Prospective Epidemiological Study of Myocardial Infarction) study been found to be associated with a reduced CHD risk for two populations in France and Northern Ireland ${ }^{(1)}$. An inverse association was likewise seen in the present study for both men and women with the highest intake of citrus fruits; however, in the adjusted models, the results were no longer statistically significant.

Juice intake was found to be associated with a $4 \%$ higher risk of ACS among women. As mentioned previously, juice intake was in the Nurses' Health Study associated with a higher risk of diabetes mellitus, which is a strong risk factor for CHD. We excluded participants with prevalent diabetes mellitus, thus presumably eliminating this as an effect modifier. However, the possibility remains that undiagnosed, preclinical diabetes mellitus in the cohort could account for the observed risk increase among women. The higher risk of diabetes mellitus seen in the Nurses' Health Study was in fact seen among women ${ }^{(10)}$. In contrast, juice intake was not associated with diabetes risk in the Nurses' Health Study II; however, the authors found that increasing intake was associated with larger weight gain, which is also a risk 
Table 3. Risk of acute coronary syndrome among men and women in the Diet, Cancer and Health cohort according to daily intake of total fruit (per $100 \mathrm{~g} / \mathrm{d}$ ), apples, citrus fruits, other fruits or juice (per $25 \mathrm{~g} / \mathrm{d})$ or by quartile $(\mathrm{Q})^{\star}$

(Incidence rate ratios (IRR) and 95\% confidence intervals)

\begin{tabular}{|c|c|c|c|c|c|c|c|c|c|c|c|c|c|c|c|c|}
\hline \multirow[b]{3}{*}{ Variable $(\mathrm{g} / \mathrm{d})$} & \multicolumn{8}{|c|}{ Men } & \multicolumn{8}{|c|}{ Women } \\
\hline & \multicolumn{2}{|r|}{ Q2 } & \multicolumn{2}{|r|}{ Q3 } & \multicolumn{2}{|r|}{ Q4 } & \multicolumn{2}{|c|}{ Linear } & \multicolumn{2}{|r|}{ Q2 } & \multicolumn{2}{|r|}{ Q3 } & \multicolumn{2}{|r|}{ Q4 } & \multicolumn{2}{|c|}{ Linear } \\
\hline & IRR & $95 \% \mathrm{Cl}$ & IRR & $95 \% \mathrm{Cl}$ & IRR & $95 \% \mathrm{Cl}$ & IRR & $95 \% \mathrm{Cl}$ & IRR & $95 \% \mathrm{Cl}$ & IRR & $95 \% \mathrm{Cl}$ & IRR & $95 \% \mathrm{Cl}$ & IRR & $95 \% \mathrm{Cl}$ \\
\hline \multicolumn{17}{|l|}{ Total fruit } \\
\hline Range $(g / d)$ & \multicolumn{2}{|c|}{$>50$ to $\leq 112$} & \multicolumn{2}{|c|}{$>112$ to $\leq 188$} & \multicolumn{2}{|r|}{$>188$} & \multicolumn{2}{|c|}{ Per $100 \mathrm{~g} / \mathrm{d}$} & \multicolumn{2}{|c|}{$>95$ to $\leq 168$} & \multicolumn{2}{|c|}{$>168$ to $\leq 272$} & \multicolumn{2}{|c|}{$>272$} & \multicolumn{2}{|c|}{ Per $100 \mathrm{~g} / \mathrm{d}$} \\
\hline Crude model $\dagger$ & 0.85 & $0.70,1.02$ & 0.72 & $0.60,0.88$ & 0.75 & $0.62,0.91$ & 0.92 & $0.86,0.97$ & 0.76 & $0.55,1.05$ & 0.70 & $0.50,0.99$ & 0.60 & $0.42,0.85$ & 0.87 & $0.80,0.96$ \\
\hline Multivariable model $1 \neq$ & 0.96 & $0.80,1.16$ & 0.88 & $0.72,1.08$ & 0.93 & $0.75,1.14$ & 0.96 & $0.90,1.02$ & 0.95 & $0.68,1.33$ & 0.93 & $0.65,1.32$ & $0 \cdot 81$ & $0.55,1.18$ & 0.93 & $0.85,1.01$ \\
\hline Multivariable model $2 \S$ & 0.98 & $0.81,1.18$ & 0.89 & $0.73,1.09$ & 0.93 & $0 \cdot 75,1 \cdot 14$ & 0.96 & $0.91,1.02$ & 0.94 & $0.67,1.32$ & 0.92 & $0.65,1.30$ & 0.80 & $0.54,1.17$ & 0.93 & $0.85,1.01$ \\
\hline \multicolumn{17}{|l|}{ Apples } \\
\hline Range $(g / d)$ & \multicolumn{2}{|c|}{$>18$ to $\leq 98$} & \multicolumn{2}{|c|}{$>98$} & \multicolumn{2}{|r|}{ - } & \multicolumn{2}{|c|}{ Per $25 \mathrm{~g} / \mathrm{d}$} & \multicolumn{2}{|c|}{$>18$ to $\leq 54$} & \multicolumn{2}{|r|}{$>54$} & & - & & $r 25 \mathrm{~g} / \mathrm{d}$ \\
\hline Crude model $\dagger$ & 0.80 & $0.67,0.94$ & 0.69 & $0.58,0.83$ & - & - & 0.95 & $0.93,0.98$ & 0.91 & $0.67,1.23$ & 0.68 & $0.50,0.92$ & - & - & 0.94 & $0.90,0.98$ \\
\hline Multivariable model $1 \ddagger$ & 0.89 & $0.75,1.05$ & 0.78 & $0.64,0.94$ & - & - & 0.97 & $0.94,0.99$ & 1.17 & $0.86,1.60$ & 0.94 & $0.67,1.30$ & - & - & 0.97 & $0.93,1.01$ \\
\hline Multivariable model $2 \S$ & 0.89 & $0.75,1.06$ & 0.78 & $0.64,0.95$ & - & - & 0.97 & $0.94,0.99$ & $1 \cdot 16$ & $0.85,1.59$ & 0.93 & $0.67,1.29$ & - & - & 0.97 & $0.93,1.01$ \\
\hline Citrus fruits & & & & & & & & & & & & & & & & \\
\hline Range $(g / d)$ & & to $\leq 11$ & $>1$ & to $\leq 43$ & & $>43$ & & $\mathrm{r} 25 \mathrm{~g} / \mathrm{d}$ & & to $\leq 19$ & $>1$ & 9 to $\leq 54$ & & $>54$ & & $r 25 \mathrm{~g} / \mathrm{d}$ \\
\hline Crude model† & 0.86 & $0.71,1.04$ & 0.75 & $0.62,0.92$ & 0.82 & $0.68,1.00$ & 0.97 & $0.93,1.02$ & 0.57 & $0.40,0.82$ & 0.73 & $0.52,1.01$ & 0.63 & $0.44,0.89$ & 0.95 & $0.89,1.01$ \\
\hline Multivariable model $1 \ddagger$ & 0.94 & $0.77,1.14$ & 0.86 & $0.70,1.06$ & 1.02 & $0.82,1.26$ & 1.00 & $0.95,1.05$ & 0.73 & $0.51,1.05$ & 1.00 & $0.71,1.41$ & 0.89 & $0.61,1.31$ & 0.98 & $0.92,1.05$ \\
\hline Multivariable model $2 \S$ & 0.96 & $0.79,1.16$ & 0.87 & $0.71,1.07$ & 1.00 & $0.81,1.24$ & 0.99 & $0.95,1.04$ & 0.71 & $0.49,1.02$ & 0.98 & $0.70,1.38$ & 0.85 & $0.58,1.25$ & 0.98 & $0.92,1.04$ \\
\hline Other fruits & & & & & & & & & & & & & & & & \\
\hline Range $(g / d)$ & & to $\leq 34$ & $>3$ & 4 to $\leq 72$ & & $>72$ & & $\mathrm{r} 25 \mathrm{~g} / \mathrm{d}$ & & to $\leq 61$ & $>6$ & to $\leq 117$ & & $>117$ & & $r 25 \mathrm{~g} / \mathrm{d}$ \\
\hline Crude model $\dagger$ & 0.76 & $0.63,0.93$ & 0.74 & $0.61,0.90$ & 0.82 & $0.68,0.99$ & 0.99 & $0.96,1.02$ & 0.85 & $0.61,1.18$ & 0.77 & $0.64,0.91$ & 0.80 & $0.67,0.96$ & 0.97 & $0.93,1.01$ \\
\hline Multivariable model $1 \ddagger$ & 0.88 & $0.72,1.07$ & 0.89 & $0.73,1.09$ & 1.05 & $0.85,1.29$ & 1.02 & $0.99,1.05$ & 0.96 & $0.68,1.34$ & 0.83 & $0.58,1.19$ & 1.03 & $0.71,1.48$ & 0.99 & $0.95,1.04$ \\
\hline Multivariable model $2 \S$ & 0.89 & $0.73,1.08$ & 0.90 & $0.74,1 \cdot 10$ & 1.07 & $0.87,1.32$ & 1.02 & $0.99,1.05$ & 0.97 & $0.70,1.36$ & 0.84 & $0.58,1.20$ & 1.05 & $0.73,1.51$ & 0.99 & $0.95,1.04$ \\
\hline Fruit juice & & & & & & & & & & & & & & & & \\
\hline Range $(g / d)$ & & to $\leq 8$ & & to $\leq 43$ & & $>43$ & & $r 25 \mathrm{~g} / \mathrm{d}$ & & to $\leq 8$ & $>\varepsilon$ & to $\leq 43$ & & $>43$ & & $r 25 \mathrm{~g} / \mathrm{d}$ \\
\hline Crude model $\dagger$ & 0.85 & $0.68,1.06$ & 0.77 & $0.64,0.91$ & 0.80 & $0.67,0.96$ & 0.99 & $0.96,1.03$ & 0.68 & $0.44,1.04$ & 0.73 & $0.53,1.00$ & 0.76 & $0.56,1.04$ & 1.03 & $0.99,1.08$ \\
\hline Multivariable model $1 \ddagger$ & 1.02 & $0.81,1.28$ & 0.93 & $0.78,1.12$ & 1.03 & $0.85,1.24$ & 1.01 & $0.98,1.05$ & 0.89 & $0.57,1.36$ & 0.92 & $0.66,1.27$ & 1.01 & $0.73,1.40$ & 1.04 & $1.00,1.09$ \\
\hline Multivariable model $2 \S$ & 1.01 & $0.81,1.27$ & 0.92 & $0.77,1.10$ & 1.03 & $0.85,1.24$ & 1.01 & $0.98,1.04$ & 0.87 & $0.56,1.34$ & 0.90 & $0.65,1.24$ & 1.01 & $0.73,1.41$ & 1.04 & $1.00,1.08$ \\
\hline
\end{tabular}

${ }^{*} \mathrm{Q} 1$ is the reference group $(\mathrm{IRR}=1)$ and is not shown. Tertiles were used for apples.

†Crude model: IRR among consumers.

Multivariable model 1: mutually adjusted and adjusted for potential confounders - BMI, length of school education (low, medium, high), smoking (never, former, current (1-14, 15-25, $>25 \mathrm{~g}$ tobacco per d)), alcohol intake (linear spline with a boundary at $10 \mathrm{~g} / \mathrm{d}$ ), alcohol abstainers and physical activity ( $\leq$ or $>3.5 \mathrm{~h}$ per week and non-active), intake of saturated fats $(\mathrm{g} / \mathrm{d})$ and whole grains $(\mathrm{g} / \mathrm{d})$.

$\S$ Multivariable model 2: as model 1 and further adjusted for total cholesterol and systolic blood pressure. 
Table 4. Risk of acute coronary syndrome among men and women in the Diet, Cancer and Health cohort according to daily intake of total vegetables (per $100 \mathrm{~g} / \mathrm{d}$ ), cruciferous or other vegetables (per $25 \mathrm{~g} / \mathrm{d}$ ) or by quartile $(\mathrm{Q})^{\star}$

(Incidence rate ratios (IRR) and $95 \%$ confidence intervals)

\begin{tabular}{|c|c|c|c|c|c|c|c|c|c|c|c|c|c|c|c|c|}
\hline & \multicolumn{8}{|c|}{ Men } & \multicolumn{8}{|c|}{ Women } \\
\hline & \multicolumn{2}{|r|}{ Q2 } & \multicolumn{2}{|r|}{ Q3 } & \multicolumn{2}{|r|}{ Q4 } & \multicolumn{2}{|c|}{ Linear } & \multicolumn{2}{|r|}{ Q2 } & \multicolumn{2}{|r|}{ Q3 } & \multicolumn{2}{|r|}{ Q4 } & \multicolumn{2}{|c|}{ Linear } \\
\hline & IRR & $95 \% \mathrm{Cl}$ & IRR & $95 \% \mathrm{Cl}$ & IRR & $95 \% \mathrm{Cl}$ & IRR & $95 \% \mathrm{Cl}$ & IRR & $95 \% \mathrm{Cl}$ & IRR & $95 \% \mathrm{Cl}$ & IRR & $95 \% \mathrm{Cl}$ & IRR & $95 \% \mathrm{Cl}$ \\
\hline \multicolumn{17}{|l|}{ Total vegetables } \\
\hline Range $(g / d)$ & \multicolumn{2}{|c|}{$>96$ to $\leq 148$} & \multicolumn{2}{|c|}{$>148$ to $\leq 211$} & \multicolumn{2}{|r|}{$>211$} & \multicolumn{2}{|c|}{ Per $100 \mathrm{~g} / \mathrm{d}$} & \multicolumn{2}{|c|}{$>110$ to $\leq 167$} & \multicolumn{2}{|c|}{$>167$ to $\leq 237$} & \multicolumn{2}{|c|}{$>237$} & \multicolumn{2}{|c|}{ Per $100 \mathrm{~g} / \mathrm{d}$} \\
\hline Crude model $\dagger$ & 0.90 & $0.75,1.08$ & 0.77 & $0.64,0.93$ & 0.64 & $0.52,0.78$ & 0.83 & $0.76,0.90$ & 0.87 & $0.64,1.19$ & 0.57 & $0.40,0.82$ & 0.60 & $0.42,0.85$ & 0.77 & $0.67,0.89$ \\
\hline Multivariable model $1 \ddagger$ & 1.06 & $0.88,1.27$ & 1.01 & $0.83,1.23$ & 0.91 & $0.73,1 \cdot 13$ & 0.97 & $0.88,1.06$ & $1 \cdot 15$ & $0.84,1.58$ & 0.88 & $0.61,1.28$ & 1.05 & $0.71,1.55$ & 0.96 & $0.83,1.11$ \\
\hline Multivariable model $2 \S$ & 1.06 & $0.88,1.28$ & 1.03 & $0.85,1.26$ & 0.93 & $0.75,1.16$ & 0.98 & $0.90,1.07$ & 1.15 & $0.84,1.58$ & 0.88 & $0.61,1.28$ & 1.09 & $0.74,1.61$ & 0.97 & $0.84,1.13$ \\
\hline \multicolumn{17}{|l|}{ Cruciferous vegetables } \\
\hline Range $(g / d)$ & \multicolumn{2}{|c|}{$>7$ to $\leq 14$} & \multicolumn{2}{|c|}{$>14$ to $\leq 24$} & \multicolumn{2}{|r|}{$>24$} & \multicolumn{2}{|c|}{ Per $25 \mathrm{~g} / \mathrm{d}$} & \multicolumn{2}{|c|}{$>8$ to $\leq 16$} & \multicolumn{2}{|c|}{$>16$ to $\leq 25$} & \multicolumn{2}{|r|}{$>25$} & \multicolumn{2}{|c|}{ Per $25 \mathrm{~g} / \mathrm{d}$} \\
\hline Crude model $\dagger$ & 0.81 & $0.67,0.98$ & 0.79 & $0.66,0.96$ & 0.78 & $0.64,0.94$ & 0.86 & $0.75,0.98$ & 0.83 & $0.60,1 \cdot 16$ & 0.80 & $0.58,1.11$ & 0.68 & $0.48,0.97$ & 0.73 & $0.57,0.94$ \\
\hline Multivariable model $1 \ddagger$ & 0.97 & $0.80,1.19$ & 1.03 & $0 \cdot 84,1 \cdot 26$ & 1.09 & $0.87,1.36$ & 1.06 & $0.92,1.23$ & 1.08 & $0.76,1.52$ & $1 \cdot 14$ & $0.80,1.62$ & $1 \cdot 10$ & $0.74,1.66$ & 0.98 & $0.75,1.28$ \\
\hline Multivariable model $2 \S$ & 0.98 & $0.81,1 \cdot 19$ & 1.03 & $0.84,1.26$ & 1.08 & $0.87,1.35$ & 1.05 & $0.91,1.22$ & 1.09 & $0.77,1.54$ & $1 \cdot 15$ & $0.80,1.64$ & $1 \cdot 12$ & $0.75,1.68$ & 0.99 & $0.76,1.29$ \\
\hline \multicolumn{17}{|l|}{ Other vegetables } \\
\hline Range $(\mathrm{g} / \mathrm{d})$ & \multicolumn{2}{|c|}{$>84$ to $\leq 131$} & \multicolumn{2}{|c|}{$>131$ to $\leq 188$} & \multicolumn{2}{|r|}{$>188$} & & $25 \mathrm{~g} / \mathrm{d}$ & $>97$ & to $\leq 149$ & $>1$ & 9 to $\leq 215$ & & $>215$ & & $r 25 \mathrm{~g} / \mathrm{d}$ \\
\hline Crude model† & 0.86 & $0.72,1.03$ & 0.77 & $0.64,0.92$ & 0.62 & $0.51,0.76$ & 0.98 & $0.97,0.99$ & 0.86 & $0.63,1.17$ & 0.54 & $0.38,0.78$ & 0.58 & $0.40,0.82$ & 0.97 & $0.96,0.99$ \\
\hline Multivariable model $1 \ddagger$ & 0.99 & $0.82,1.19$ & 0.97 & $0.79,1.19$ & 0.84 & $0.66,1.07$ & 0.99 & $0.98,1.00$ & $1 \cdot 13$ & $0.82,1.56$ & 0.84 & $0.57,1.25$ & 1.02 & $0.67,1.56$ & 0.99 & $0.98,1.01$ \\
\hline Multivariable model $2 \S$ & 0.99 & $0.82,1.20$ & 0.99 & $0.81,1.22$ & 0.87 & $0.68,1.10$ & 1.00 & $0.99,1.01$ & $1 \cdot 12$ & $0.81,1.54$ & 0.85 & $0.57,1.25$ & 1.04 & $0.68,1.60$ & 1.00 & $0.98,1.02$ \\
\hline
\end{tabular}

${ }^{*} \mathrm{Q} 1$ is the reference group $(\mathrm{IRR}=1)$ and is not shown.

† Crude model: IRR among consumers.

(lod (linear spline with a boundary at $10 \mathrm{~g} / \mathrm{d}$ ), alcohol abstainers and physical activity ( $\leq$ or $>3.5 \mathrm{~h}$ per week and non-active), intake of saturated fats $(\mathrm{g} / \mathrm{d})$ and whole grains $(\mathrm{g} / \mathrm{d})$.

§Multivariable model 2: as model 1 and further adjusted for total cholesterol and systolic blood pressure. 
factor of $\mathrm{CHD}^{(24)}$. Furthermore, it might be that juice intake is associated with intake of other dietary factors that we did not correct for and thus represents an association with some other lifestyle or dietary factor.

In conclusion, the present study provides some support for an inverse association between a higher intake of fruit, specifically apples, and reduced risk of ACS. However, more research is needed to examine the biological mechanisms behind this association. Biomarker studies, such as metabolomics, would provide a novel and more accurate way of assessing disease risk in association with dietary intakes.

\section{Acknowledgements}

The present study was supported by the ISAFRUIT project, which is funded by the European Commission under the Thematic Priority 5 - Food Quality and Safety of the 6th Framework Programme of research and technological development (RTD; contract no. FP6-FOOD-CT-2006016279). The Danish Diet, Cancer and Health cohort study was approved by the regional ethical committees of human studies in Copenhagen and Aarhus and by the Danish Data Protection Agency.

The views and opinions expressed in the present publication are purely those of the writers and may not in any circumstances be regarded as stating an official position of the European Commission.

The contributions of the authors were as follows: A. T. and K. O. collected the data; L. H. was the principal investigator and performed data analysis, wrote the manuscript and is the guarantor; L. O. D., A. O., J. C., A. T., E. B. S. and K. O. contributed to data analysis and to the writing of the manuscript.

There are no conflicts of interest.

\section{References}

1. Dauchet L, Ferrieres J, Arveiler D, et al. (2004) Frequency of fruit and vegetable consumption and coronary heart disease in France and Northern Ireland: the PRIME study. Br J Nutr 92, 963-972.

2. Joshipura KJ, Hu FB, Manson JE, et al. (2001) The effect of fruit and vegetable intake on risk for coronary heart disease. Ann Intern Med 134, 1106-1114.

3. Liu S, Lee IM, Ajani U, et al. (2001) Intake of vegetables rich in carotenoids and risk of coronary heart disease in men: The Physicians' Health Study. Int J Epidemiol 30, 130-135.

4. Hirvonen T, Pietinen P, Virtanen M, et al. (2001) Intake of flavonols and flavones and risk of coronary heart disease in male smokers. Epidemiology 12, 62-67.

5. Liu S, Manson JE, Lee IM, et al. (2000) Fruit and vegetable intake and risk of cardiovascular disease: the Women's Health Study. Am J Clin Nutr 72, 922-928.

6. Dauchet L, Amouyel P, Hercberg S, et al. (2006) Fruit and vegetable consumption and risk of coronary heart disease: a meta-analysis of cohort studies. J Nutr 136, 2588-2593.
7. He FJ, Nowson CA, Lucas M, et al. (2007) Increased consumption of fruit and vegetables is related to a reduced risk of coronary heart disease: meta-analysis of cohort studies. $J$ Hum Hypertens 21, 717-728.

8. Lampe JW (1999) Health effects of vegetables and fruit: assessing mechanisms of action in human experimental studies. Am J Clin Nutr 70, 475S-490S.

9. Nagasako-Akazome Y, Kanda T, Ikeda M, et al. (2005) Serum cholesterol-lowering effect of apple polyphenols in healthy subjects. J Oleo Sci 54, 143-151.

10. Bazzano LA, Li TY, Joshipura KJ, et al. (2008) Intake of fruit, vegetables, and fruit juices and risk of diabetes in women. Diabetes Care 31, 1311-1317.

11. Cornelis MC, El-Sohemy A \& Campos H (2007) GSTT1 genotype modifies the association between cruciferous vegetable intake and the risk of myocardial infarction. $A m$ J Clin Nutr 86, 752-758.

12. Haraldsdottir J, Tjonneland A \& Overvad K (1994) Validity of individual portion size estimates in a food frequency questionnaire. Int J Epidemiol 23, 786-796.

13. Tjonneland A, Overvad K, Haraldsdottir J, et al. (1991) Validation of a semiquantitative food frequency questionnaire developed in Denmark. Int J Epidemiol 20, 906-912.

14. Tjonneland A, Olsen A, Boll K, et al. (2007) Study design, exposure variables, and socioeconomic determinants of participation in Diet, Cancer and Health: a population-based prospective cohort study of 57053 men and women in Denmark. Scand J Public Health 35, 432-441.

15. Joensen AM, Jensen MK, Overvad K, et al. (2009) Predictive values of acute coronary syndrome discharge diagnoses differed in the Danish National Patient Registry. J Clin Epidemiol 62, 188-194.

16. Lauritsen J (2007) FoodCalc. http://www.ibt.ku.dk/jesper/ foodcalc/ (accessed February 2009).

17. Greenland S (1995) Avoiding power loss associated with categorization and ordinal scores in dose-response and trend analysis. Epidemiology 6, 450-454.

18. Knekt P, Jarvinen R, Reunanen A, et al. (1996) Flavonoid intake and coronary mortality in Finland: a cohort study. BMJ 312, 478-481.

19. Arts IC, Jacobs DR Jr, Harnack LJ, et al. (2001) Dietary catechins in relation to coronary heart disease death among postmenopausal women. Epidemiology 12, 668-675.

20. Aprikian O, Levrat-Verny MA, Besson C, et al. (2001) Apple favourably affects parameters of cholesterol metabolism and of anti-oxidative protection in cholesterol-fed rats. Food Chem 75, 445-452.

21. Bobek P, Ginter E, Jurcovicova M, et al. (1990) Effect of dehydrated apple products on the serum and liver lipids in Syrian hamsters. Nahrung 34, 783-789.

22. Osada K, Suzuki T, Kawakami Y, et al. (2006) Dose-dependent hypocholesterolemic actions of dietary apple polyphenol in rats fed cholesterol. Lipids 41, 133-139.

23. Sable-Amplis R, Sicart R \& Agid R (1983) Further studies on the cholesterol-lowering effect of apple in humans: biochemical mechanisms involved. Nutr Res 3, 325-328.

24. Schulze MB, Manson JE, Ludwig DS, et al. (2004) Sugarsweetened beverages, weight gain, and incidence of type 2 diabetes in young and middle-aged women. JAMA 292, 927-934. 